\title{
Thermodynamics of dense hadronic matter in a parity doublet model
}

\author{
Chihiro Sasaki ${ }^{1}$ and Igor Mishustin ${ }^{1,2}$ \\ ${ }^{1}$ Frankfurt Institute for Advanced Studies, D-60438 Frankfurt am Main, Germany \\ ${ }^{2}$ Kurchatov Institute, Russian Research Center, Moscow 123182, Russia
}

(Dated: October 23, 2018)

\begin{abstract}
We study thermodynamics of nuclear matter in a two-flavored parity doublet model within the mean field approximation. Parameters of the model are chosen to reproduce correctly the properties of the nuclear ground state. The model predicts two phase transitions in nuclear matter, a liquidgas phase transition at normal nuclear density and a chiral transition at higher density. At finite temperature the pion decay constant exhibits a considerable reduction at intermediate values of chemical potential, which is traced back to the presence of the liquid-gas transition, and approaches zero at higher chemical potential associated with the chiral symmetry restoration. A "transition" from meson-rich to baryon-rich matter is also discussed.
\end{abstract}

PACS numbers: 21.65.-f, 12.39.Fe, 21.65.Mn

\section{INTRODUCTION}

Model studies of dense quark matter have suggested a rich phase structure of QCD at temperatures and quark chemical potentials of order $\Lambda_{\mathrm{QCD}}$. Our knowledge on the phase structure however remains limited and the description of strongly interacting matter does not reach a consensus yet [1]. In particular, properties of baryons near the chiral symmetry restoration are poorly understood. We believe that the realistic modeling of dense baryonic matter must take into account the existence of the nuclear matter saturation point, i.e. the bound state at baryon density $\rho_{0}=0.16 \mathrm{fm}^{-3}$, like in Walecka type models [2]. Several chiral models with pure hadronic degrees of freedom [3, 4] have been constructed in such a way that the nuclear matter has the true ground state. Other models consider a nucleon as a dynamical boundstate of a diquark and a quark [5].

In chiral models it is mostly assumed that a constituent quark transforms under chiral rotations as a bare quark. According to this naive assignment, dynamical chiral symmetry breaking entirely generates a baryon mass which thus vanishes at the restoration. According to an alternative, mirror assignment [6, 7], dynamical chiral symmetry breaking generates a mass difference between parity partners and the chiral symmetry restoration does not necessarily dictate the chiral partners to be massless. Mirror baryons embedded in linear and non-linear chiral Lagrangians have been applied to study their phenomenology in vacuum [6-8], nuclear matter 9, 10] and neutron starts 11, although it remains an open question which scenario is preferred by nature. Identifying the true parity partner of a nucleon is also an issue. In the mirror models $N(1535)$ is usually taken to be the negative parity state. This choice however fails to reproduce the decay width to a nucleon and $\eta$. This might indicate another negative parity state lighter than the $N(1535)$ [10], which has not been observed so far.

In this paper we apply the parity doublet model to a hot and dense hadronic matter and study the phase structure of a chiral phase transition as well as a liquid- gas transition of nuclear matter. The main interest is the phase boundary of the chiral symmetry restoration on top of the nuclear matter ground state. A light negative parity partner will be considered as a phenomenological option and we show how the phase boundary lines are affected. The phase transitions in large number of colors are also discussed.

\section{PARITY DOUBLET MODEL}

The negative-parity nucleon field is introduced such that it transforms in the opposite way as the positive parity nucleon under $\mathrm{SU}(2)_{L} \times \mathrm{SU}(2)_{R}[6]$ :

$$
\begin{aligned}
& \psi_{+L} \rightarrow L \psi_{+L}, \quad \psi_{+R} \rightarrow R \psi_{+R}, \\
& \psi_{-L} \rightarrow R \psi_{-L}, \quad \psi_{-R} \rightarrow L \psi_{-R},
\end{aligned}
$$

with $L \in \mathrm{SU}(2)_{L}$ and $R \in \mathrm{SU}(2)_{R}$, and this allows a chirally invariant mass term. The Lagrangian of the mirror baryons is given by

$$
\begin{aligned}
\mathcal{L}= & \bar{\psi}_{+} i \not \partial \psi_{+}+\bar{\psi}_{-} i \not \partial \psi_{-}+m_{0}\left(\bar{\psi}_{-} \gamma_{5} \psi_{+}-\bar{\psi}_{+} \gamma_{5} \psi_{-}\right) \\
& +a \bar{\psi}_{+}\left(\sigma+i \gamma_{5} \vec{\tau} \cdot \vec{\pi}\right) \psi_{+}+b \bar{\psi}_{-}\left(\sigma-i \gamma_{5} \vec{\tau} \cdot \vec{\pi}\right) \psi_{-} \\
& -g_{\omega} \bar{\psi}_{+} \gamma_{\mu} \omega^{\mu} \psi_{+}-g_{\omega} \bar{\psi}_{-} \gamma_{\mu} \omega^{\mu} \psi_{-}+\mathcal{L}_{M},
\end{aligned}
$$

where $a, b$ and $g_{\omega}$ are the coupling constants of the mesons to the baryons. The mesonic part follows the standard linear sigma model Lagrangian,

$$
\begin{aligned}
\mathcal{L}_{M}= & \frac{1}{2} \partial_{\mu} \sigma \cdot \partial^{\mu} \sigma+\frac{1}{2} \partial_{\mu} \vec{\pi} \cdot \partial^{\mu} \vec{\pi}-\frac{1}{4} F_{\mu \nu} F^{\mu \nu} \\
& +\frac{1}{2} m_{\omega}^{2} \omega_{\mu} \omega^{\mu}+g_{4}^{4}\left(\omega_{\mu} \omega^{\mu}\right)^{2} \\
& +\frac{1}{2} \bar{\mu}^{2}\left(\sigma^{2}+\vec{\pi}^{2}\right)-\frac{1}{4} \lambda\left(\sigma^{2}+\vec{\pi}^{2}\right)^{2}+\epsilon \sigma(2.3)
\end{aligned}
$$

with the field strength tensor of the omega meson, $F_{\mu \nu}=$ $\partial_{\mu} \omega_{\nu}-\partial_{\nu} \omega_{\mu}$. The parameters $\lambda, \bar{\mu}$ and $\epsilon$ are related to the sigma and pion masses and the pion decay constant 
in vacuum as

$$
\begin{aligned}
\lambda & =\frac{m_{\sigma}^{2}-m_{\pi}^{2}}{2 \sigma_{0}^{2}}, \\
\bar{\mu}^{2} & =\frac{m_{\sigma}^{2}-3 m_{\pi}^{2}}{2}, \\
\epsilon & =m_{\pi}^{2} f_{\pi},
\end{aligned}
$$

with $m_{\pi}=138 \mathrm{MeV}, f_{\pi}=93 \mathrm{MeV}$ and the vacuum expectation value of the sigma field $\sigma_{0}=f_{\pi}$. The vacuum mass of the omega field is $m_{\omega}=783 \mathrm{MeV}$. Other mesonic parameters, $m_{\sigma}$ and $g_{4}$, as well as the chiral invariant mass $m_{0}$ will be fixed to reproduce the known properties of nuclear matter.

The mass term of (2.2) is diagonalized by the mass eigenstates of the parity doubled nucleons, $N_{+}$and $N_{-}$ via

$$
\left(\begin{array}{l}
N_{+} \\
N_{-}
\end{array}\right)=\frac{1}{\sqrt{2 \cosh \delta}}\left(\begin{array}{cc}
e^{\delta / 2} & \gamma_{5} e^{-\delta / 2} \\
\gamma_{5} e^{-\delta / 2} & -e^{\delta / 2}
\end{array}\right)\left(\begin{array}{l}
\psi_{+} \\
\psi_{-}
\end{array}\right)
$$

with $\sinh \delta=-(a+b) \sigma / 2 m_{0}$. The masses of $N_{ \pm}$are thus given by

$$
m_{N \pm}=\frac{1}{2}\left[\sqrt{(a+b)^{2} \sigma^{2}+4 m_{0}^{2}} \mp(a-b) \sigma\right] .
$$

When the chiral symmetry is restored $\left(\sigma_{0}=0\right)$, the two states become degenerate, $m_{N+}=m_{N-}=m_{0}$. In contrast, in the naive transformation under chiral symmetry, i.e. $\psi_{ \pm L} \rightarrow L \psi_{ \pm L}$ and $\psi_{ \pm R} \rightarrow R \psi_{ \pm R}$, the nucleon mass is generated solely by the spontaneous chiral symmetry breaking and thus vanishes with its restoration.

Applying a mean field approximation ${ }^{\# 1}$, the thermodynamic potential is

$$
\begin{aligned}
\Omega= & \sum_{i= \pm} \gamma_{i} \int \frac{d^{3} \vec{p}}{(2 \pi)^{3}} T\left[\ln \left(1-n_{f}\left(T, \mu^{*} ; m_{i}\right)\right)\right. \\
& \left.+\ln \left(1-\bar{n}_{f}\left(T, \mu^{*} ; m_{i}\right)\right)\right] \\
& -\frac{1}{2} m_{\omega}^{2} \omega_{0}^{2}-g_{4}^{4} \omega_{0}^{4}-\frac{1}{2} \bar{\mu}^{2} \sigma^{2}+\frac{1}{4} \lambda \sigma^{4}-\epsilon \sigma
\end{aligned}
$$

where $\gamma_{i}$ denotes the spin-isospin degeneracy factor of a nucleon and $n_{f}\left(\bar{n}_{f}\right)$ is the Fermi-Dirac distribution function for a fermion (an anti-fermion) defined by

$$
\begin{aligned}
& n_{f}=\frac{1}{1+e^{\left(E_{i}-\mu^{*}\right) / T}}, \\
& \bar{n}_{f}=\frac{1}{1+e^{\left(E_{i}+\mu^{*}\right) / T}},
\end{aligned}
$$

with the energy $E_{i}=\sqrt{\vec{p}^{2}+m_{i}^{2}}$ and the effective chemical potential $\mu^{*}=\mu-g_{\omega} \omega_{0}$. Imposing the stationary

\#1 Rotational invariance requires $\langle\vec{\omega}\rangle=0$ and only the timecomponent $\left\langle\omega_{0}\right\rangle$ does not vanish.

\begin{tabular}{ccc}
\hline & set A & set B \\
\hline$m_{N-}[\mathrm{MeV}]$ & 1500 & 1200 \\
$m_{0}[\mathrm{MeV}]$ & 790 & 790 \\
$m_{\sigma}[\mathrm{MeV}]$ & 370.63 & 318.56 \\
$g_{\omega}$ & 6.79 & 6.08 \\
$a$ & 13.00 & 9.16 \\
$b$ & 6.97 & 6.35 \\
$\bar{\mu}[\mathrm{MeV}]$ & 199.26 & 147.50 \\
$\lambda$ & 6.82 & 4.75 \\
\hline
\end{tabular}

TABLE I: Set of parameters with $g_{4}=0$ [10]. The vacuum nucleon mass is $m_{N+}=935 \mathrm{MeV}$.

condition, $\partial \Omega / \partial \sigma=\partial \Omega / \partial \omega_{0}=0$, one obtains the coupled field equations:

$$
\begin{aligned}
& \sum_{i= \pm} \gamma_{i} \int \frac{d^{3} \vec{p}}{(2 \pi)^{3}} \frac{m_{i}}{E_{i}} \frac{\partial m_{i}}{\partial \sigma}\left[n_{f}+\bar{n}_{f}\right]-\bar{\mu}^{2} \sigma+\lambda \sigma^{3}-\epsilon=0, \\
& g_{\omega} \sum_{i= \pm} \gamma_{i} \int \frac{d^{3} \vec{p}}{(2 \pi)^{3}}\left[n_{f}-\bar{n}_{f}\right]-m_{\omega}^{2} \omega_{0}-4 g_{4}^{4} \omega_{0}^{3}=0 .(2.9)
\end{aligned}
$$

The net baryon number density is given by

$$
\rho=\sum_{i= \pm} \gamma_{i} \int \frac{d^{3} \vec{p}}{(2 \pi)^{3}}\left[n_{f}-\bar{n}_{f}\right]
$$

The model parameters are fixed to reproduce the nuclear matter saturation properties at $T=0$ :

$$
\begin{aligned}
& E / A\left(\rho_{0}\right)-m_{N+}=-16 \mathrm{MeV}, \\
& \rho_{0}=0.16 \mathrm{fm}^{-3} .
\end{aligned}
$$

Originally, the negative parity state has been chosen to be $N(1535)[\underline{6}, 7]$. However this choice is incompatible with the width of decay into $\eta N$. Also, the mass difference between $N(1535)$ and $N_{+}$is about $560 \mathrm{MeV}$, that is well above the QCD scale $\Lambda_{\mathrm{QCD}} \sim 200 \mathrm{MeV}$. The spontaneous chiral symmetry breaking might not play much role on the physics of such excited states. Another candidate, which is lighter and has a stronger coupling to the pion, is considered to be a phenomenological option [10, 11]. We will thus consider two sets of model parameters as given in [10] (see Table 【): With these sets of parameters the incompressibility defined by

$$
K=\left.9 \rho_{0}^{2} \frac{\partial^{2}(E / A)}{\partial \rho^{2}}\right|_{\rho_{0}}=\left.9 \frac{\partial P}{\partial \rho}\right|_{\rho_{0}}=\left.9 \rho_{0} \frac{\partial \mu_{B}}{\partial \rho}\right|_{\rho_{0}},
$$

is $430-510 \mathrm{MeV}$. This is slightly higher but still compatible to the suggested range, $200-400 \mathrm{MeV}$, from the analysis of giant mono-pole resonances [12]. Note that finite $g_{4}$ decreases the incompressibility, e.g. $K=440 \mathrm{MeV}$ $\left(m_{N-}=1500 \mathrm{MeV}\right)$ and $K=370 \mathrm{MeV}\left(m_{N-}=1200\right.$ $\mathrm{MeV}$ ) for $g_{4}=3.8$ [10]. This however does not change much either the (pseudo)critical density or the order of phase transitions. For simplicity of our calculations, we will therefore take $g_{4}=0$. 


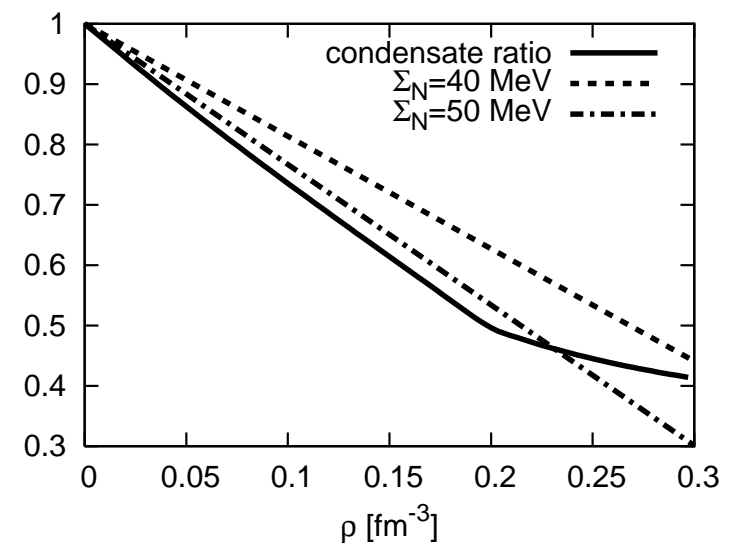

FIG. 1: The ratio of the $\bar{q} q$ condensate in medium to its vacuum value. The calculation was done at $T=0$ with the parameter set A. For set B there is no significant difference from the above result.

\section{THERMODYNAMICS OF THE MODEL}

The density dependence of the quark condensate $\langle\bar{q} q\rangle$ can be extracted using the Feynman-Hellmann theorem and it is model independent to the leading order in nucleon density [13],

$$
\frac{\langle\bar{q} q\rangle_{\rho}}{\langle\bar{q} q\rangle_{\mathrm{vac}}}=1-\frac{\Sigma_{N}}{m_{\pi}^{2} f_{\pi}^{2}} \rho,
$$

with the nucleon sigma term $\Sigma_{N}=45 \pm 8 \mathrm{MeV}$ [14]. Fig. 1 shows a comparison of the low-energy theorem (3.1) with the in-medium quark condensate calculated in the parity doublet model. The model describes the correct linearity at low densities up to around the saturation density $\rho_{0}$. The value of the condensate, however, decreases somewhat stronger than Eq. (3.1). This can be considered as an artifact of our simple model and taking other parameters (with non-vanishing $g_{4}$ ) or more systematic treatment would make a better agreement with the empirical value of $\Sigma_{N}$. In-medium chiral condensate has been explored in chiral perturbation theory constrained by the nuclear matter properties and shown to have a strong pion mass dependence, and a substantial deviation from the linear density approximation was found already at $\rho \sim 0.2 \mathrm{fm}^{-3}$ where the ratio is $\sim 0.7$ [15]. In that framework the two-pion exchange correlations with virtual $\Delta(1232)$ resonances are responsible for stabilizing the quark condensate, which are not incorporated in the current mean field model.

It is remarkable that the parity doublet model at $T=0$ describes a liquid-gas transition of nuclear matter and a first-order (set A) or crossover type (set B) of chiral symmetry restoration [10]. At an intermediate temperature, above these phase transitions, the order parameter typically shows a double-step structure as shown in Fig. 2] The first drop in $\langle\sigma\rangle$ appears much below the

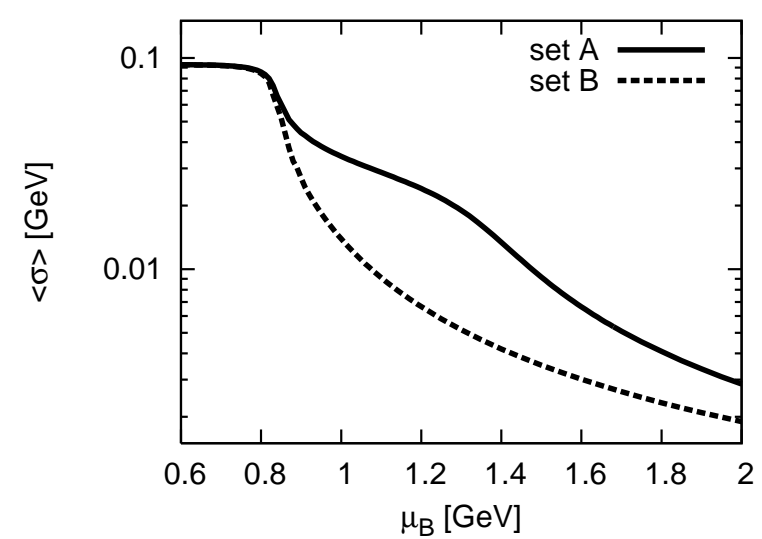

FIG. 2: The expectation value of the sigma field as a function of baryon chemical potential at $T=50 \mathrm{MeV}$.

chiral crossover and this is traced back to the remnant of the liquid-gas phase transition. The second drop in $\langle\sigma\rangle$ happens at a higher $\mu_{B}$ associated with the chiral symmetry restoration. The two-step structure is observed only at rather low temperatures, and at high temperatures $\langle\sigma\rangle$ eventually experiences a single reduction due to the chiral crossover. Note, as mentioned above, the first reduction will weaken when improvements are included to reproduce Eq. (3.1).

In the parity doublet model one can deal with inmedium mesons and baryons explicitly. Besides the baryon masses given in Eq. (2.6), we define the effective meson masses in matter by

$$
M_{\sigma}^{2}=\frac{\partial^{2} \Omega}{\partial \sigma^{2}}, \quad M_{\vec{\pi}}^{2}=\frac{\partial^{2} \Omega}{\partial \vec{\pi}^{2}}, \quad M_{\omega}^{2}=-\frac{\partial^{2} \Omega}{\partial \omega_{0}^{2}},
$$

where $\Omega$ is given in Eq. (2.7). The total meson number density is given by

$$
\rho_{M}=\sum_{i=\sigma, \pi, \omega_{0}} \gamma_{i} \int \frac{d^{3} \vec{p}}{(2 \pi)^{3}} n_{b}\left(T ; M_{i}\right)
$$

where $\gamma_{i}$ is the corresponding mesonic degeneracy factors, and $n_{b}$ is the Bose-Einstein distribution function,

$$
n_{b}=\frac{1}{e^{E_{i} / T}-1}
$$

with the corresponding particle energy $E_{i}=\sqrt{\vec{p}^{2}+M_{i}^{2}}$. For simplicity, here we consider only $\sigma, \pi$ and $\omega$ mesons, although other mesons, especially $\rho$ with $\gamma_{\rho}=9$ may somewhat contribute too. The meson density depends on temperature and it is instructive to compare it with the baryon density. We consider the ratio of the meson to the baryon number density,

$$
\frac{\rho_{M}}{\rho_{B}}=1
$$


to determine a trajectory in the $T-\mu_{B}$ plane. This trajectory separates a meson-dominated region from a baryon-dominated one and characterizes a meson-tobaryon "transition" in dense matter. This is similar to the definition adopted in Ref. [16] where the statistical model parameters $T$ and $\mu_{B}$ were extracted from the hadron yield ratios. While the statistical model successfully describes the particle abundances at chemical freeze-out, it cannot handle the chiral phase transition. In the parity doublet model, on the other hand, the nuclear matter ground state is built in and the chiral symmetry restoration is also described. Thus, the ratio (3.5) can be used to find the critical points on the phase diagram within a self-consistent chiral approach.

In Fig. 3 we show the phase diagram of the parity doublet model for the two parameter sets discussed above. At zero temperature the system experiences a first-order liquid-gas transition at $\mu_{B}=923 \mathrm{MeV}$ and the baryon density shows a jump from zero to a finite value $\rho \neq 0$. The order of chiral phase transition and its location depend on the set of parameters, especially on mass of the negative parity state [10]. Roughly speaking this phase transition occurs when the baryon chemical potential reaches the mass of the negative parity state, $\mu_{B} \sim m_{N-}$. If we take the most frequently used value $m_{N-}=1500$ $\mathrm{MeV}$, then in addition to the nuclear liquid-gas phase transition we obtain a weak first-order chiral transition at $\rho \sim 10 \rho_{0}$. With a lower mass $m_{N-}=1200 \mathrm{MeV}$ we get no true chiral phase transition but only a crossover at much lower density $\rho \sim 3 \rho_{0} \# 2$. The liquid-gas transition survives up to $T=27 \mathrm{MeV}$. Above this temperature there is no sharp phase transition but the order parameter is still attracted by the critical point, corresponding to the first drop in $\langle\sigma\rangle$. This makes an additional crossover line terminating at the liquid-gas critical point. Another crossover line corresponding to the chiral symmetry restoration follows the steepest descent of the second reduction in $\langle\sigma\rangle$. With increasing temperature the two crossover lines become closer and finally merge. The former crossover line, associated with a trace of the liquid-gas phase transition, is not well determined from the susceptibility when two bumps are getting closer to each other.

In contrast, the trajectory defined in Eq. (3.5) is basically driven by the density effect with the hadron masses being not far from their vacuum values. The line is almost independent of the parameter set and goes rather close to the liquid-gas transition line. The chiral crossover and the meson-baryon transition lines intersect at $\left(T, \mu_{B}\right) \sim(150,450) \mathrm{MeV}$. The parity doublet model thus describes 3 domains: a chirally broken phase with mesons thermodynamically dominating, an-

\#2 Finite $g_{4}=3.8$, which gives a slightly better fit for the incompressibility $K$, does not change its order, but makes a first-order chiral transition somewhat stronger [10]. other chirally broken phase where baryons are more dominant and the chirally restored phase, which can be identified with quarkyonic matter [17, 18]. It is worthy to note that this point is fairly close to the estimated triple point at which hadronic matter, quarkyonic matter and quark-gluon plasma may coexist [16].

\section{CHIRAL CROSSOVER IN NUCLEONIC NJL MODEL}

There exist other chiral models which have attempted to describe nuclear matter ground state [3, 44]. The vector-type interactions at finite density, isoscalar vector and a mixed scalar-vector ones, play a significant role to reproduce correctly the nuclear saturation point. Fermions in those works were introduced in the naive assignment and the nucleon thus becomes massless in the chiral limit when chiral symmetry is restored. In this section we give a brief discussion about the thermodynamics at finite temperatures of a nucleonic Nambu-JonaLasinio model (NJL) [4] and compare to the result in the parity doublet model.

At $\mu_{B}=0$ the vector interaction does not affect the gap equation,

$$
m_{N}=m_{N}^{0}+\gamma_{N} G_{S} \int \frac{d^{3} p}{(2 \pi)^{3}} \frac{m_{N}}{E}\left[1-2 n_{f}\left(m_{N} ; T\right)\right]
$$

where $m_{N}^{0}$ denotes explicit symmetry breaking mass and $G_{S}$ the strength of scalar four-fermion interaction. Another parameter is a momentum cutoff $\Lambda$ which regularizes the integral in Eq. (4.1). The parameters fixed to reproduce Eqs. (2.11) and (2.12) at zero temperature are given to be $\Lambda=0.4 \mathrm{GeV}, G_{S}=1.677 \mathrm{GeVfm}^{3}$ and $m_{N}^{0}=41.3 \mathrm{MeV}$ [4]. At $T=0$ in addition to the nuclear liquid-gas transition this model also predicts the chiral crossover-type transition at $\rho \sim 3 \rho_{0}$. Here we check the prediction of this model at finite $T$. Assuming a secondorder chiral transition in the chiral limit, $m_{N} \rightarrow 0$ for $m_{N}^{0}=0$, the gap equation determines the critical temperature as

$$
\begin{aligned}
& \frac{\pi^{2}}{12} T^{2}+T^{2} L_{2}\left[-e^{-\Lambda / T}\right]-T \Lambda \ln \left(1+e^{-\Lambda / T}\right) \\
& =\frac{\Lambda^{2}}{8}-\frac{\pi^{2}}{2 \gamma_{N} G_{S}}
\end{aligned}
$$

where $L_{2}[z]=\sum_{n=1}^{\infty} z^{n} / n^{2}$ is Euler's dilogarithm. Substituting the parameter values one finds for the critical temperature $T_{c}=450 \mathrm{MeV}$. Numerical calculation with explicit breaking leads to a crossover with slightly higher temperature $T_{c}=510 \mathrm{MeV}$. Obviously this is too high to be accepted as a reliable critical temperature compatible with the Lattice QCD predictions [19]. On the other hand, this $T_{c}$ is above the cutoff and therefore the model can not be used near the transition region. Although larger $\Lambda$ can cure this drawback, another problem ap- 

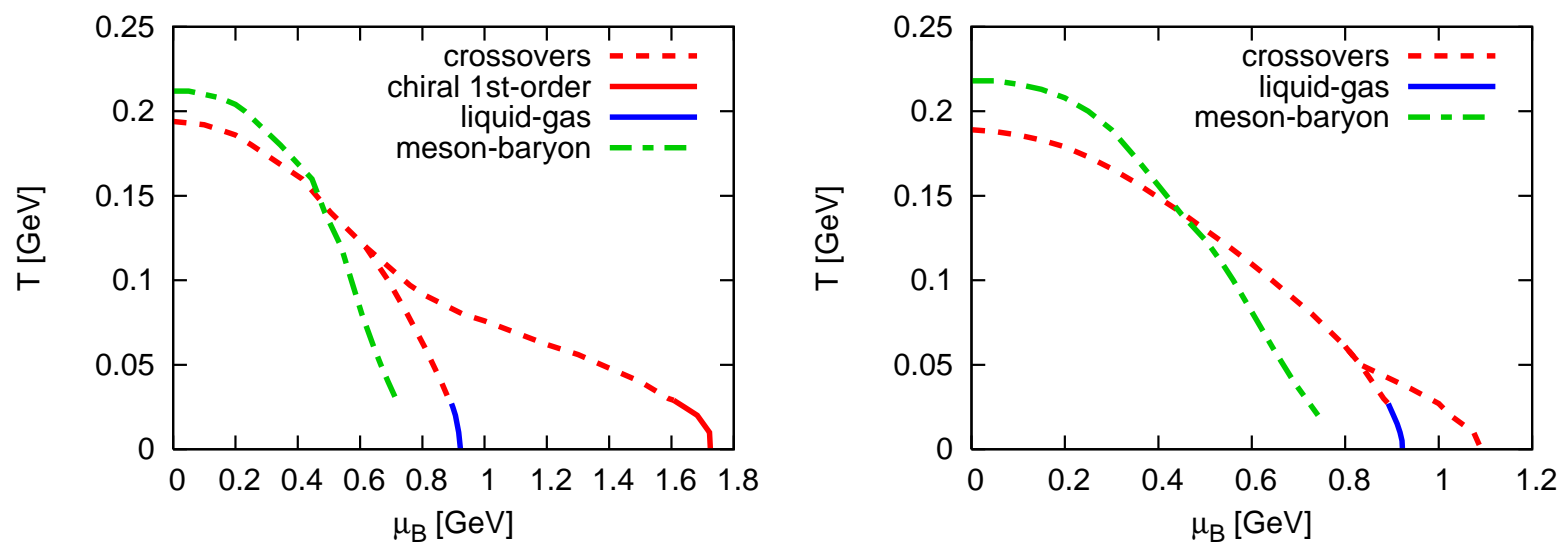

FIG. 3: The phase diagram for the set A (left) and for the set B (right). The crossovers correspond to the local/global maxima in the order parameter susceptibility.

pears, i.e. the bare nucleon mass becomes lower than three times the bare quark mass [4].

In contrast, the parity doublet model leads to $T_{c} \sim 190$ $\mathrm{MeV}$ at which the nucleon and its negative-parity partner remain massive $m_{N+} \sim m_{N_{-}} \sim m_{0}$. Therefore, this model satisfies the relevant phenomenological constraints not only on nuclear matter properties but also on the chiral crossover at small chemical potential in a reasonable way.

\section{PHASE STRUCTURE FOR LARGE $N_{c}$}

An interesting issue to address is how the phase structure is modified by increasing the number of colors $N_{c}$. In large $N_{c}$ limit baryons arise as a soliton in the Skyrme model picture. In the current hadronic model we deal with the baryons as elementary particles, and thus there is no direct access to its dynamical description in the large $N_{c}$ limit, where microscopic structure of the baryon is particularly important. Besides, it still remains uncertain at which scale a Skyrmion collapses (see e.g. Ref. [20] and references therein). Nevertheless, it is worthwhile to study a tendency for various $N_{c}$, which may partly bridge a gap between the real and large $N_{c}$ QCD.

Following Ref. [21] we adopt the $N_{c}$ dependences in the model parameters as

$$
\begin{aligned}
& m_{N \pm} \rightarrow\left(N_{c} / 3\right) m_{N \pm}, \quad m_{0} \rightarrow\left(N_{c} / 3\right) m_{0}, \\
& a \rightarrow \sqrt{N_{c} / 3} a, \quad b \rightarrow \sqrt{N_{c} / 3} b \\
& g_{\omega} \rightarrow \sqrt{N_{c} / 3} g_{\omega}, \quad f_{\pi} \rightarrow \sqrt{N_{c} / 3} f_{\pi}, \\
& \lambda \rightarrow\left(3 / N_{c}\right) \lambda,
\end{aligned}
$$

and the meson masses and the parameter $\bar{\mu}$ do not carry $N_{c}$ dependences. Inserting these scaling factors into the gap equations, one can perform thermodynamical calculations. In the following, without loss of generality, we take zero temperature and consider the liquid-gas tran-

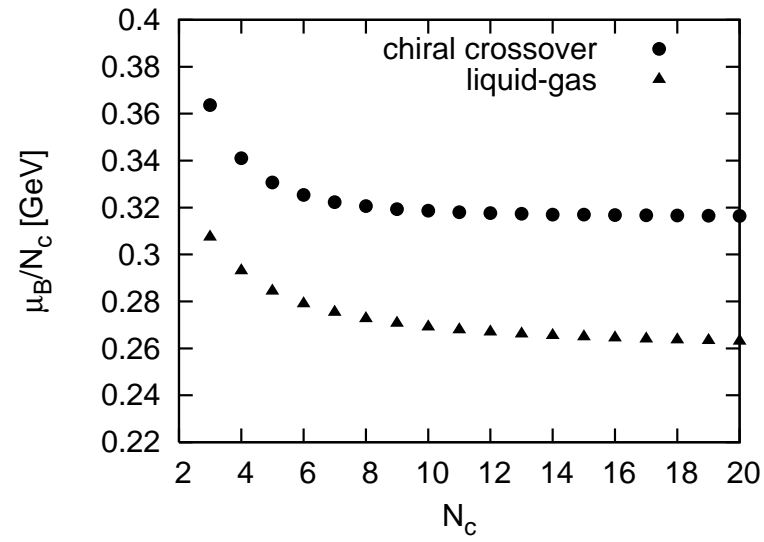

FIG. 4: The (pseudo)critical chemical potentials for chiral (circle) and liquid-gas (triangle) for various $N_{c}$. The negative parity state was taken to be $N_{-}(1200)$.

sition and chiral symmetry restoration in cold nuclear matter.

As seen in section 3, how far the two transitions are separated essentially relies on the mass of the negative parity state: The liquid-gas transition from vacuum to nuclear matter ground state happens roughly at $\mu_{B} \sim m_{N+}$. When its parity partner is taken to be $N(1535)$, the initial mass gap between the parity partners, which is generated by the spontaneous chiral symmetry breaking, is rather large $\sim 560 \mathrm{MeV}$. Compared with the case taking $N(1200)$, more matter compression is required in order to decrease the gap and consequently to restore the chiral symmetry. This mass gap between the parity partners scales linearly in $N_{c}$ and therefore no coincidence of the liquid-gas and chiral phase transitions is generally expected. In fact, the actual calculation in this model follows this consideration, as shown in Fig. 4. With increasing $N_{c}$ the two phase transitions are system- 
atically shifted toward higher chemical potentials. One sees the linear- $N_{c}$ behavior in $\mu_{B}$ for around $N_{c}>10$. On the other hand, if the effective coupling constants increase less slowly with $N_{c}$ as discussed in Ref. [20], the two phase transitions may coincide in the large $N_{c}$ limit [22]. More microscopic view of the baryons at large $N_{c}$ might change this prediction, but this study is beyond the scope of this paper.

Deconfinement dynamics which is not dealt with in the current model might also modify the above result, especially the phase boundaries at higher temperatures. In fact, a pure NJL model for constituent quarks predicts a chiral phase boundary of elliptic shape for any $N_{c}$. While, NJL models with Polyakov loops (PNJL) 23] describes a hierarchy in quark and gluon sectors with respect to $N_{c}$ so that the deconfinement phase transition always happens at the critical value in a pure gluon system, $T_{0} \sim 280$ $\mathrm{MeV}$ [17].

\section{CONCLUSIONS}

We have investigated thermodynamics of nuclear matter in a parity doublet model within the mean field approximation. The model describes the nuclear matter ground state at zero temperature and a chiral crossover at zero chemical potential at a reasonable temperature, which are the minimal requirements to describe the QCD thermodynamics. The first-order phase transitions appear only at low temperatures, below $T \sim 30 \mathrm{MeV}$. Nevertheless, at higher temperature they still affect the order parameter which exhibits a substantial decrease near the liquid-gas and chiral transitions. If the chiral symmetry restoration is of first order, criticality around the end points of the two first-order phase transitions will be the same due to the identical universality class [24].

The trace of the nuclear liquid-gas transition is mostly overlapped with the meson-to-baryon "transition" characterizing the ratio of the particle number densities, $\rho_{M} / \rho_{B}=1$. Its trajectory has only a weak parameterdependence and the intersection to the chiral crossover appears at $\left(T, \mu_{B}\right) \sim(150,450) \mathrm{MeV}$. The chiral phase boundary at lower temperature, on the other hand, is strongly affected by the negative parity state. The heavier parity partner induces a larger baryon-rich domain in Nambu-Goldstone (NG) phase and thus a strong enhancement in the baryon density is not necessarily accompanied by the chiral symmetry restoration. The true parity partner of the nucleon needs to be identified. This requires to solve the model dynamically, since in NG phase the chiral symmetry does not dictate any nontrivial relations between the masses of parity partners and their couplings [25].

When increasing $N_{c}$ the two phase transitions are shifted to higher chemical potentials and remain separated. This tendency is similar to the observation in the PNJL model [17]: The threshold at which the baryon number density becomes finite and a first-order chiral phase transition do not coincide for any $N_{c}$ but are close with each other. This could indicate that the quarkyonic matter [18] is realized in the chirally restored phase with confinement. However, this seems to be unlikely, without introducing any gapless modes in a Wigner-Weyl phase, since chiral anomalies in low-energy theories do not match with those in QCD [26]. Further investigations along with the trace anomaly, which is also responsible for appearance of a scale in QCD, may bridge the thermodynamics of the toy model used in this paper to the origin of $m_{0}$.

\section{Acknowledgments}

We acknowledge stimulating discussions with M. Lombardo, K. Redlich, L. M. Satarov, S. Schramm, G. Torrieri and W. Weise. This work has been partly supported by the Hessian LOEWE initiative through the Helmholtz International Center for FAIR (HIC for FAIR), and by the grants NS-7235.2010.2 and RFBR 09-02-91331 (Russia). C.S. thanks the organizers of the programme "New Frontiers in QCD" for their kind hospitality at Yukawa Institute for Theoretical Physics.
[1] C. Sasaki, Nucl. Phys. A 830, 649C (2009), and references therein.

[2] B. D. Serot and J. D. Walecka, Int. J. Mod. Phys. E 6, 515 (1997).

[3] J. Boguta, Phys. Lett. B 120, 34 (1983), I. Mishustin, J. Bondorf and M. Rho, Nucl. Phys. A 555, 215 (1993), G. W. Carter and P. J. Ellis, Nucl. Phys. A 628, 325 (1998), P. Papazoglou, S. Schramm, J. SchaffnerBielich, H. Stoecker and W. Greiner, Phys. Rev. C 57, 2576 (1998), P. Papazoglou, D. Zschiesche, S. Schramm, J. Schaffner-Bielich, H. Stoecker and W. Greiner, Phys. Rev. C 59, 411 (1999).

[4] V. Koch, T. S. Biro, J. Kunz and U. Mosel, Phys. Lett. B 185, 1 (1987), M. Buballa, Nucl. Phys. A 611, 393
(1996), I. N. Mishustin, L. M. Satarov and W. Greiner, Phys. Rept. 391, 363 (2004).

[5] W. Bentz and A. W. Thomas, Nucl. Phys. A 696, 138 (2001), W. Bentz, T. Horikawa, N. Ishii and A. W. Thomas, Nucl. Phys. A 720, 95 (2003).

[6] C. E. Detar and T. Kunihiro, Phys. Rev. D 39, 2805 (1989).

[7] Y. Nemoto, D. Jido, M. Oka and A. Hosaka, Phys. Rev. D 57, 4124 (1998), D. Jido, Y. Nemoto, M. Oka and A. Hosaka, Nucl. Phys. A 671, 471 (2000), D. Jido, T. Hatsuda and T. Kunihiro, Phys. Rev. Lett. 84, 3252 (2000), D. Jido, M. Oka and A. Hosaka, Prog. Theor. Phys. 106, 873 (2001).

[8] S. Gallas, F. Giacosa and D. H. Rischke, arXiv:0907.5084 
[hep-ph].

[9] T. Hatsuda and M. Prakash, Phys. Lett. B 224, 11 (1989).

[10] D. Zschiesche, L. Tolos, J. Schaffner-Bielich and R. D. Pisarski, Phys. Rev. C 75, 055202 (2007).

[11] V. Dexheimer, S. Schramm and D. Zschiesche, Phys. Rev. C 77, 025803 (2008), V. Dexheimer, G. Pagliara, L. Tolos, J. Schaffner-Bielich and S. Schramm, Eur. Phys. J. A 38, 105 (2008).

[12] J. P. Blaizot, Phys. Rept. 64, 171 (1980), D. Vretenar, T. Niksic and P. Ring, Phys. Rev. C 68, 024310 (2003), D. H. Youngblood, Y. W. Lui, H. L. Clark, B. John, Y. Tokimoto and X. Chen, Phys. Rev. C 69, 034315 (2004).

[13] T. D. Cohen, R. J. Furnstahl and D. K. Griegel, Phys. Rev. C 45, 1881 (1992).

[14] J. Gasser, H. Leutwyler and M. E. Sainio, Phys. Lett. B 253, 252 (1991).

[15] N. Kaiser, P. de Homont and W. Weise, Phys. Rev. C 77, 025204 (2008).

[16] A. Andronic et al., Nucl. Phys. A 837, 65 (2010).

[17] L. McLerran, K. Redlich and C. Sasaki, Nucl. Phys. A 824, 86 (2009).
[18] L. McLerran and R. D. Pisarski, Nucl. Phys. A 796, 83 (2007), Y. Hidaka, L. D. McLerran and R. D. Pisarski, Nucl. Phys. A 808, 117 (2008).

[19] M. Cheng et al., Phys. Rev. D 81, 054510 (2010), S. Borsanyi, Z. Fodor, C. Hoelbling, S. D. Katz, S. Krieg, C. Ratti and K. K. Szabo [Wuppertal-Budapest Collaboration], arXiv:1005.3508 [hep-lat].

[20] Y. Hidaka, T. Kojo, L. McLerran and R. D. Pisarski, arXiv:1004.2261 [hep-ph].

[21] E. Witten, Nucl. Phys. B 160, 57 (1979).

[22] G. Torrieri and I. Mishustin, paper in preparation.

[23] K. Fukushima, Phys. Lett. B 591, 277 (2004), C. Ratti, M. A. Thaler and W. Weise, Phys. Rev. D 73, 014019 (2006).

[24] A. M. Halasz, A. D. Jackson, R. E. Shrock, M. A. Stephanov and J. J. M. Verbaarschot, Phys. Rev. D 58, 096007 (1998).

[25] R. L. Jaffe, D. Pirjol and A. Scardicchio, Phys. Rev. Lett. 96, 121601 (2006); Phys. Rev. D 74, 057901 (2006); Phys. Rept. 435, 157 (2006).

[26] C. Sasaki, arXiv:0910.4375 [hep-ph]; arXiv:1004.5299 [hep-ph]. 